Dieses Dokument ist eine Zweitveröffentlichung (Postprint Version) / This is a self-archiving document (accepted version):

Adrian Ehrenhofer, Manfred Hahn, Martin Hofmann, Thomas Wallmersperger

Mechanical behavior and pore integration density optimization of switchable hydrogel composite membranes

Erstveröffentlichung in / First published in:

Journal of Intelligent Material Systems and Structures. 2020, 31 (3), S. 425 - 435. SAGE Journals. ISSN 1530-8138.

DOI: https://doi.org/10.1177/1045389X19888802

Diese Version ist verfügbar / This version is available on:

https://nbn-resolving.org/urn:nbn:de:bsz:14-qucosa2-742116 


\title{
Mechanical behavior and pore integration density optimization of switchable hydrogel composite membranes
}

\author{
Adrian Ehrenhofer' , Manfred Hahn',2, Martin Hofmann' and Thomas \\ Wallmersperger'
}

\begin{abstract}
Switchable hydrogel-layered composite membranes can be used for the analysis of particle size distributions. This functionality is provided by pores with controllable diameter. In order to obtain a device that can be used to measure the cell size distribution in native biological samples, lots of switchable pores are required. In the current work, we model and simulate the mechanical behavior of active composite membranes with switchable pores. This is done in order to find the maximum number of pores that can be integrated into a membrane without cross-influencing effects on the actuation of the pores. Therefore, we investigate $(I)$ the interaction of active pores inside the multifunctional composite and (2) the membrane bending under microfluidic pressure load. We show that through miniaturization, sufficient pores can be added to a permeation control membrane for processing native blood samples. The envisioned device allows a parallelized measurement of cell sizes in a simple lab-on-a-chip setup.
\end{abstract}

\section{Keywords}

Active composite membranes, hydrogels, modeling and simulation, cell sorting, microfluidics

\section{Introduction: membranes with switchable permeability}

Hydrogel layer systems can be used for switchable filtration in microfluidic systems when the diameter of pores for filtration is precisely controlled. In order to obtain such a system with tunable pore sizes, we designed and fabricated a membrane composite system in our previous studies (Ehrenhofer et al., 2016a, 2016b). The active system is based on a polyethylene terephthalate (PET) backbone membrane with circular apertures with diameter $d^{\text {aperture }}=290 \mu \mathrm{m}$. The membrane is surface polymerized with poly( $N$-isopropylacrylamide) (PNiPAAm), which is a temperature-responsive hydrogel (Gerlach and Arndt, 2009). In this active composite, pore structures of different shapes can be inserted (see Figure 1).

When a microfluidic particle-laden flow is sent through the membrane, particles are filtered due to size exclusion (see Figure 2). The goal of the current work is the mechanical characterization and optimization of this system.

The separation and concentration of cells in water can be performed using fluorescent or magnetic labels (fluorescent-activated cell sorting (FACS)/magneticactivated cell sorting (MACS)) or label-free (Shields et al., 2015). The approach which is presented in our previous studies (Ehrenhofer et al., 2016a) is based on a membrane with switchable permeability and is therefore label-free. In similar approaches from literature, switchable membranes are constructed, for example, by filling membrane pores with hydrogel and by changing the permeability of the porous medium (Adrus and Ulbricht, 2012). The smart material class of hydrogels offers the advantage of switchability through different

\footnotetext{
'Institut für Festkörpermechanik, Technische Universität Dresden, Dresden, Germany

${ }^{2}$ Wilhelm Büchner Hochschule, Pfungstadt, Germany
}

\section{Corresponding authors:}

Adrian Ehrenhofer, Institut für Festkörpermechanik, Technische Universität Dresden, 01062 Dresden, Germany. Email: adrian.ehrenhofer@tu-dresden.de

Thomas Wallmersperger, Institut für Festkörpermechanik, Technische Universität Dresden, 01062 Dresden, Germany.

Email: thomas.wallmersperger@tu-dresden.de 


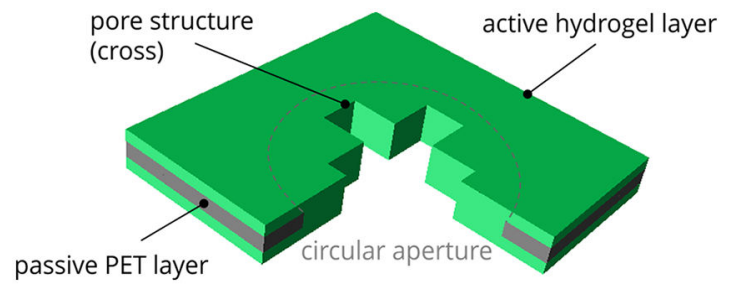

Figure I. Schematic layout of the composite system in the environment of a single cross-shaped pore structure. The dashed line represents the round aperture in the backbone membrane, that is, the passive PET layer.

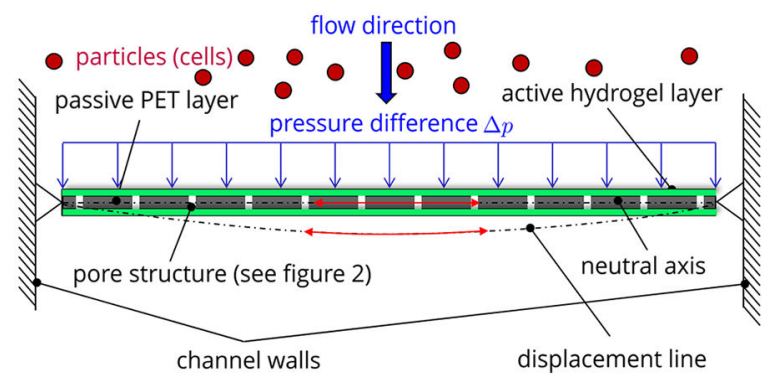

Figure 2. Setup of the membrane system with multiple pores inside the microfluidic channel. The particle-laden flow passes the permeation control membrane in downward direction. Dependent on the activation stimulus, the pore structures change their size and thus their filtration properties.

stimuli like temperature, salt concentration, or pH-value (Ehrenhofer et al., 2018; Gerlach and Arndt, 2009). Hydrogels can be used as actuators (Attaran et al., 2015) or sensors (Binder et al., 2017).

The mechanical behavior of composites comprising hydrogels can be described through modeling and simulation. The active hydrogel behavior is described, for example, by the multifield theory (Sobczyk and Wallmersperger, 2016; Wallmersperger et al., 2013) or the theory of porous media (Acartürk, 2009; Bluhm et al., 2016; Ehlers, 1996; Leichsenring and Wallmersperger, 2017; Li, 2010). The mechanical behavior of hydrogels is experimentally obtained and described, for example, in the publications of Anseth et al. (1996) or Seuss et al. (2016). Layer systems with active surfaces are described by Kang and Huang (2011) or Sun and Qing (2011). The passive part of our system can be described with well-known composite theories (Mota Soares et al., 1999). Homogenization for scale transition is described, for example, by Forest (2002).

In the current section, we describe the design goal for the permeation control membrane. Then, the composition of the system and its mechanical treatment are described in the "Modeling of active composite membranes" section. The "Results and discussion" section features the simulation results and their discussion regarding the pore integration density (PID) and miniaturization. The conclusions are drawn in the "Conclusion and outlook" section.

\section{I.I. Setup of the permeation control membrane and design goal}

The analysis of the flow through switchable pores leads to the conclusion that the flux drop is characteristic: for every (temperature-dependent) opening state of a pore, the difference between particle-free and blocked state can be derived (Ehrenhofer and Wallmersperger, 2017a). However, this idea only works if there are enough pores inside the membrane. Since the investigated fluid-particle system is blood - which has a particle density of approximately $5 \times 10^{12} \mathrm{~L}^{-1}$ (Jelkmann, 2011) - a large number of pores is required. In analogy to the number of parts integrated into a microelectronic system, the PID gives a comparative value for this system

$$
\text { PID }=\frac{\text { Number of pores }}{\text { Area of membrane }} \stackrel{!}{\rightarrow} \text { max }
$$

The area of all pores $A_{\text {pore }}$ over the total membrane area $A^{\text {membrane }}$ (including pores) is dimensionless, and therefore more useful for engineering purposes

$$
\overline{\text { PID }}=\frac{\text { Area of pores }}{\text { Membrane area }}=\frac{A_{\text {pore }}}{A^{\text {membrane }}}
$$

The task can therefore be formulated as design optimization under the constraints of mechanical and filtration functionality. The investigated system is a hydrogel layer composite system inside a microfluidic setup. It is subjected to a pressure distribution due to the microfluidic flow. It is also subjected to internal stresses due to actuation. In the final setup, it will also be subjected to contact forces of the to-be-filtrated particles, which will be addressed in future studies.

\section{Modeling of active composite membranes}

In the mechanical engineer's understanding, membranes are thin structures with the main characteristic that their deformation behavior is not dominated by their bending stiffness. In their deformed state, the curved form leads to a tensile stress distribution, which characterizes the displacement line (Mota Soares et al., 1999). In contrast, from the process engineer's or biologist's point of view, membranes are mainly characterized by their functionality, that is, separating compartments of fluid with different physical properties as well as allowing fluid and/or particle permeation.

In the current section, we present the mechanical background of the active composite system. At first, we 


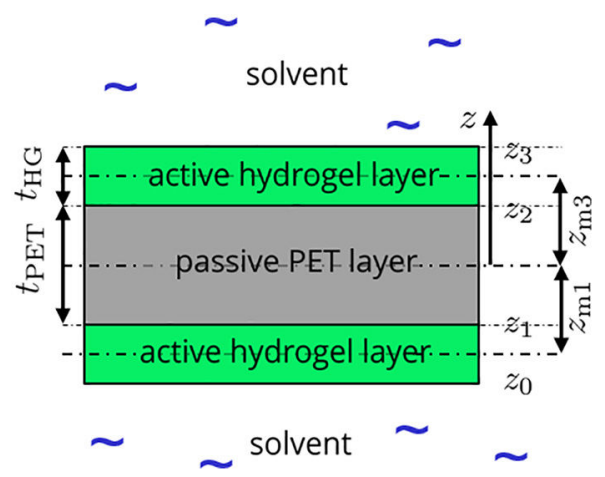

Figure 3. Setup of the layers in the composite system in a solution bath with coordinate system for the Classical Laminate Theory.

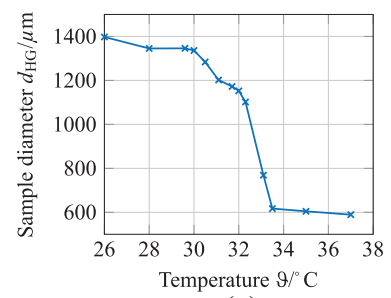

(a)

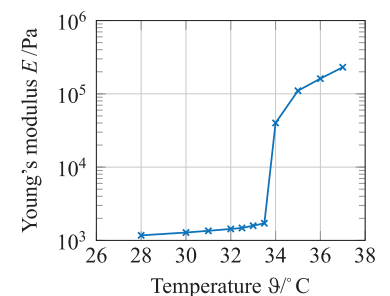

(b)
Figure 4. Properties of the temperature-sensitive hydrogel PNiPAAm in the investigated temperature range: (a) free swelling behavior of PNiPAAm sample (original data published in our previous studies; Ehrenhofer et al., 2016a) and (b) elastic properties of PNiPAAm over temperature according to the data from Seuss et al. (2016).

describe the system in the "Setup of the active composite membrane" section. The active behavior is represented using the Temperature Expansion Model (TEM) which is described in the "TEM for active behavior" section. Then, the minimal pore distance which is the core value to acquire an optimized PID is derived (see the "Determination of the minimum pore distance" section). The pattern arrangement of pores on a membrane is described in the "Pore patterns" section. The bending behavior of the membrane is discussed in the "Bending of the active composite system" section. The respective simulation results are given in the "Results and discussion" section.

\section{I. Setup of the active composite membrane}

The active system being considered in the current work is a trilayer composite consisting of two active hydrogel layers that sandwich one passive layer (see Figure 3). The passive layer is the PET backbone membrane which has a much higher stiffness in comparison to the thin active hydrogel layers at the top and bottom side. In the current section, the material behavior is described separately for each layer and in combined form.

2.I.I. Passive PET backbone layer. The middle layer of the regarded system is composed of biaxially oriented polyethylene terephthalate (BOPET; see Figure 3). This material has no active properties for the investigated stimuli ranges and acts as the stiffening backbone. The product used in the experiments is a DuPont Mylar foil. The axial Young's modulus of the BOPET layer is assumed as $E^{\mathrm{PET}} \approx 4895 \mathrm{MPa}$ according to the product data sheet.

2.I.2. Active hydrogel layers. The hydrogel layers are surface polymerized to the PET backbone layer and form the active parts of the system. When these layers are subjected to a temperature stimulus, actuation occurs due to water movement into the material (Ehrenhofer et al., 2018). In free state, temperature decrease leads to isotropic swelling (volume expansion) of the hydrogel due to water transport from the surrounding aqueous environment (solution). The respective swelling curve is presented in Figure 4(a) (Ehrenhofer et al., 2016a). In the current setup, no such free swelling occurs since the setup is constrained. This means that the mechanical constraints - that is, the connection to the backbone membrane-hinder the hydrogel from its free expansion, and therefore mechanical stresses occur. These stresses can be understood as pre-stresses for the bending that occurs due to the microfluidic pressure; however, these pre-stresses are very small in comparison to the bending stresses.

The material behavior of the hydrogel layers that are bonded to the PET backbone membrane is described using TEM. This model can be used for the swelling of a hydrogel as explained by Ehrenhofer et al. (Ehrenhofer et al. 2018; Ehrenhofer and Wallmersperger, 2017b; see the "TEM for active behavior" section). In the current work, the material behavior is the swelling of PNiPAAm. The original data were presented in Ehrenhofer et al. (2016a). There, we found excellent agreement between simulations using the TEM and the experimental data for pore opening and closing.

Please note that the hydrogel itself is a homogenized material. It represents a mixture of water and polymer. Both are incompressible but in watery environment, water and polymer form a compressible material together. The Poisson's ratio varies over the swelling degree (Urayama and Takigawa, 2012). However, the data for PNiPAAm show only a very small dependency, which is neglected in the current work. In accordance to our previous simulations (Ehrenhofer et al., 2016a), we assume a Poisson's ratio of $\nu=0.3$ for the material. 


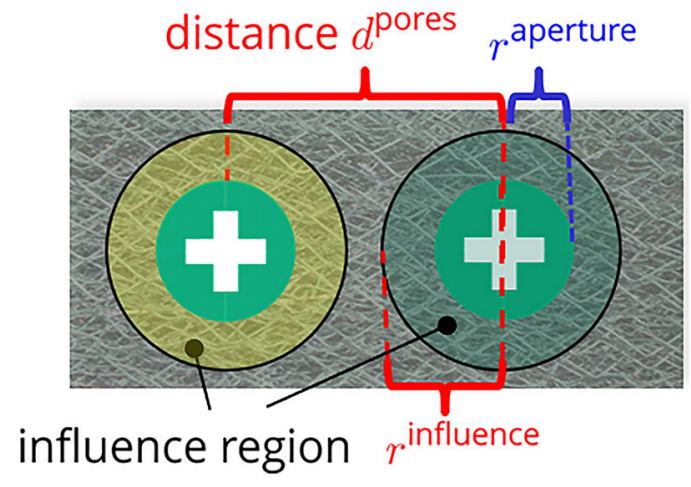

(a)

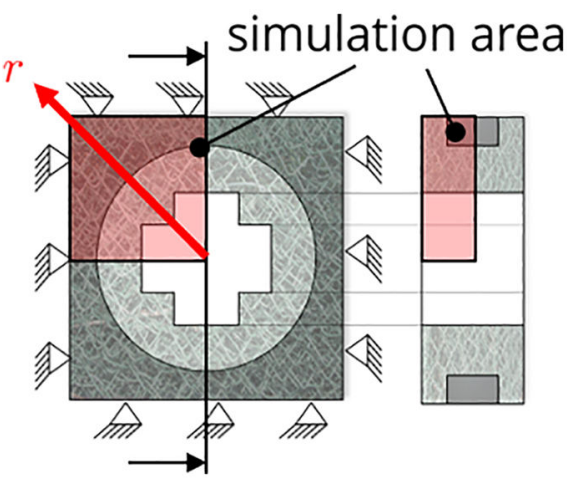

(b)

Figure 5. Influence regions for pores and definition of the pore distance. In order to allow independence of pore actuation, the influence regions should not overlap, that is, $d^{\text {pores }} \geqslant 2 r^{\text {influence }}$ : (a) influence regions and radii, and (b) simulation area of the perforated setup.

In addition, the effect of varying elastic properties over the swelling process has to be considered. The data for the Young's modulus are taken from the literature (Seuss et al., 2016) and range between $E^{\mathrm{PNiPAAm}}=1 \mathrm{kPa}$ and $230 \mathrm{kPa}$ for a temperature range of $\vartheta=28^{\circ} \mathrm{C}$ to $36^{\circ} \mathrm{C}$ (see Figure 4(b)). In order to represent a complete swelling cycle of the hydrogel i.e. lowering the temperature until the pore is completely closed, the value of $\vartheta=26^{\circ} \mathrm{C}$ is needed. Here, we assume that no more swelling occurs for temperatures much lower than the lower critical solution temperature (LCST) $\vartheta_{\text {LCST }}=32^{\circ} \mathrm{C}$. Without major swelling, the Young's modulus does not significantly change; hence, $E^{\mathrm{PNiPAAm}}\left(26^{\circ} \mathrm{C}\right)=E^{\mathrm{PNiPAAm}}\left(28^{\circ} \mathrm{C}\right)$.

\subsection{TEM for active behavior}

In our previous studies (Ehrenhofer et al., 2016a, 2018), we proposed a mechanical analogy model for the hydrogel actuation. It is based on the analogy of isotropic hydrogel swelling and thermal expansion. Comparison between simulations of the pore system and experiments has shown excellent agreement (Ehrenhofer et al. 2016a). Therefore, we apply this model in the present work to derive additional insights through simulations of the whole membrane system. The TEM can be applied within the framework of the composite theory as well, in order to allow the description of the current system.

The total Hencky strain $\varepsilon_{m n}^{\mathrm{H}}$-described by the deformation gradient $F_{k K}$-is multiplicatively decomposed into two parts: (1) the isotropic and stress-free swelling part $F^{\text {th }}$ and (2) the mechanical part $F_{k K}^{\mathrm{el}}$ (Galante et al., 2013)

$$
F_{k K}=F^{\text {th }} F_{k K}^{\mathrm{el}}
$$

For the mechanical (elastic) part $F_{k K}^{\mathrm{el}}$, linear elasticity is assumed since the swelling process is dominated by the swelling part and not by external forces. The material model therefore supposes a linear relationship between elastic strain and stress.

For the true Cauchy-stress $\sigma_{k l}$, the complete field problem is represented by

$$
\begin{aligned}
\text { Balances: } & \sigma_{k l, k}+f_{l}=0, \quad \sigma_{k l}=\sigma_{l k} \\
\text { Kinematics: } & \varepsilon_{k l}^{\mathrm{H}}=\frac{1}{2} \ln \left(B_{k l}\right) \\
\text { Material law: } & \sigma_{k l}=E_{k l m n}\left(\varepsilon_{m n}^{\mathrm{H}}-\alpha_{m n} \Delta \vartheta\right)
\end{aligned}
$$

Here, $f_{l}$ represents the external force density. $B_{k l}=F_{k K} F_{l K}$ is the left Cauchy-Green deformation tensor for finite deformations. $E_{k l m n}$ is the elasticity tensor which includes the temperature-dependent Young's modulus $E(\vartheta)$ and the constant Poisson's ratio $\nu$ according to the "Setup of the active composite membrane" section. $\alpha_{m n}(\vartheta)$ is the temperature-dependent expansion coefficient tensor $\left(\alpha_{m n}=\alpha \delta_{m n}\right.$ for isotropic material behavior), and $\Delta \vartheta$ is the temperature difference with respect to the reference state (Ehrenhofer and Wallmersperger, 2018). For the currently investigated hydrogel PNiPAAm, isotropic material behavior is assumed.

Linear-elastic material behavior and nonlinear kinematics are usually implemented in commercial finite element software. Therefore, we utilize Abaqus 2018 (version 6.14-2) for our simulations. The correct implementation of the material behavior has been verified with experimental data of free swelling in our previous studies (Ehrenhofer et al., 2016a, 2018).

\subsection{Determination of the minimum pore distance}

In order to design the composite membrane in such a way that the maximum number of pores is obtained, the minimum distance between pores has to be analyzed and numeralized. Pores can be considered independent 
if the minimal distance $d^{\text {pores, min }}$ is chosen. For this distance, all stress influences of a pore-denoted by the

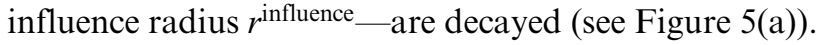

The influence region of a pore is assumed to be circular with the influence radius $r^{\text {influence }}$. It can be estimated in analogy to the Saint-Venant principle. This principle states that for a discontinuity (e.g. load or geometric discontinuity), the influence region can be estimated with a characteristic length, for example, the height of the structure or the pore diameter. However, there are exceptions like the well-known example of the perforated two-dimensional (2D) structure under stretch. This example shows a decay of the influence over three times of the hole diameter.

Therefore, numerical studies of the influence radius are necessary. The active composite plate without inserted pores is regarded as the state of reference. Then, the membrane with inserted pores is compared to this reference state to identify the influence length of the disturbance, that is, $r^{\text {influence }}$ of the pore.

The base setup - that is, the state of stress distribution without the influence of the pore - can be obtained through different ways: this is either (1) in very large distance from the pore using the complete perforated setup, (2) through a separate simulation of the composite setup without pores, or (3) through simplified theories like the Classical Laminate Theory (CLT). In the current work, we use the analytical approach which we verified with the second option since with the complete setup, it is very time intensive and therefore numerically expensive. The derivations of the describing equations of the CLT are shown in Appendix 1.

The perforated setup of the composite plate is the membrane with integrated pores. The simulation setup is depicted in Figure 5(b). Please note that the simulation region was chosen larger so that the decaying stress influence can be captured. The TEM described above was applied in Abaqus using the methods described in our previous studies (Ehrenhofer et al., 2016a; Ehrenhofer and Wallmersperger, 2018).

\subsection{Pore patterns}

In order to analyze blood samples, a large number of pores is necessary. The arrangement of pores - that is, the circles of their respective influence areas - on the membrane can be (1) in densest packings (solution of the box problem; Goldberg, 1970; Graham and Lubachevsky, 1996) and (2) in patterns. While (1) is especially beneficial for a small number of pores inside a small membrane, (2) allows an easier manufacturing process due to the regularity. Also, the large number of pores needed on the membrane makes the difference with respect to the densest packing very small.

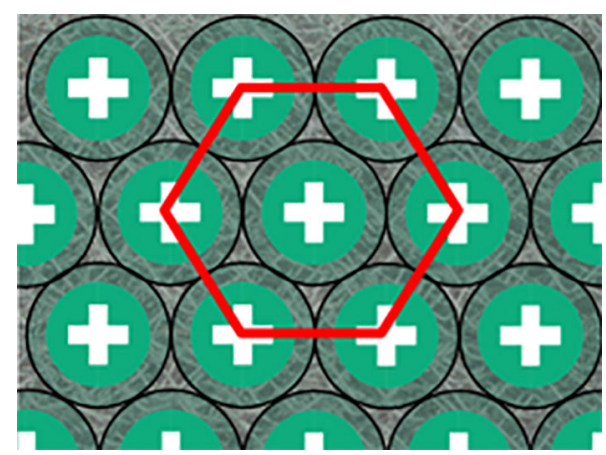

Figure 6. The hexagonal pattern is the densest twodimensional arrangement of circles in a pattern.

In the current work, we use a hexagonal pattern since it provides the densest arrangement of circles in a plane (Borchardt-Ott, 2009; see Figure 6). Other patterns are rectangular, quadratic, rhombic, or skewed, and may offer advantages if different pore sizes and forms are combined on a membrane. This will be part of our future investigations.

According to Thue's theorem (Borchardt-Ott, 2009; Chang and Wang, 2010), the ratio of area covered by circles with radius $r^{\text {influence }}$ over the total area in a hexagonal packing amounts to $\pi \sqrt{3} / 6$; hence, the PID for the setup in Figure 6 is

$$
\begin{gathered}
\overline{\mathrm{PID}}^{\text {hexagonal }}=\frac{\text { Pore influence area }}{\text { Membrane area }} \\
=\frac{\pi\left(r^{\text {influence }}\right)^{2}}{A^{\text {membrane }}}=\frac{\pi \sqrt{3}}{6} \approx 91 \%
\end{gathered}
$$

\subsection{Bending of the active composite system}

After the identification of the minimal pore distance described in the previous section, the bending of the composite system can be investigated. This is done in order to assess if the membrane deformations due to the pressure load lead to loss of function either due to widening of the pores or due to bursting of the membrane.

It can be assumed that the backbone membrane takes the role of counteracting (1) rupture and (2) mechanical deformation. This is due to the parallel trilayer setup, which leads to equal strain in the layers (membrane/disk state) as well as a linear strain distribution in thickness direction (Bernoulli's hypothesis) (see Appendix 1). Since the layers are under similar strain and the hydrogel is much softer, the mechanical stresses due to stretching and bending are much lower. Together with the high yield strain of hydrogels, this leads to the fact that (1) the PET backbone membrane ruptures earlier. Due to its low elastic modulus, (2) the hydrogel also 
does not contribute much toward the stiffness matrices $\underline{A}$ and $\underline{D}$ in the CLT. The overall setup can therefore be regarded without the soft hydrogel layers since their only function is to provide switchable permeability.

In order to assess this assumption, studies with the finite element model of a representative elemental volume were performed. They have shown that the stiffness of the PET backbone membrane governs the overall stiffness of the composite system. Therefore, it is sufficient to simulate the PET membrane with circular apertures. The circular apertures have the distance $d^{\text {pores }}=2 r^{\text {influence }}$ (Figure 5(a)) and the hexagonal pattern (Figure 6). The simulations of membrane bending are performed in the "Simulation results of the membrane bending" section. If the membrane system is further optimized, for example, by thinning the backbone membrane, that is, $t_{\mathrm{PET}} \downarrow$, this assumption no longer holds true and the elastic modulus of the overall membrane becomes direction-dependent.

Due to the simplified membrane setup - that is, only apertures without the hydrogel pore structures are regarded - a smaller area of the membrane is subjected to pressure. In order to conserve the total reaction force from the original system, which can be found by integrating the pressure, the overall pressure has to be raised. The corrected pressure $p^{\text {corrected }}$ for $N^{\text {Pores }}$ pores can be derived with the total force that amounts to

$$
\begin{aligned}
F^{\text {total }} & =p A^{\text {membrane }} \\
& =p^{\text {corrected }}\left(A^{\text {membrane }}-N^{\text {Pores }} \frac{\pi}{4} d_{\text {aperture }}^{2}\right)
\end{aligned}
$$

\section{Results and discussion}

The simulation of the minimal distance between switchable pores is performed with a single pore setup in the "Simulation of the single pore for the derivation of minimum distance" section. From these investigations, a design for a permeation control membrane with the adequate number of pores can be derived. In the "Simulation results of the membrane bending" section, the bending of a simplified membrane setup with the densest packing of pores is simulated.

\section{I. Simulation of the single pore for the derivation of minimum distance}

As explained in the "Determination of the minimum pore distance" section, the size of the influencing region can be obtained by the comparison of the perforated setup and the base setup. Therefore, a threedimensional (3D) finite element model for the crossshaped active pore is constructed using symmetry conditions (see Figure 7).

The two materials - active and passive - are implemented in accordance to the "Setup of the active composite membrane" section. The active behavior is

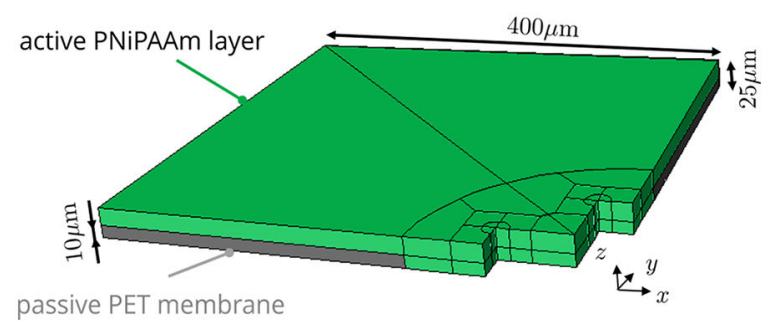

Figure 7. An eighth model of the pore setup can be used for the simulation of the influence regions due to symmetry in geometry, boundary conditions and loads for the swelling case.

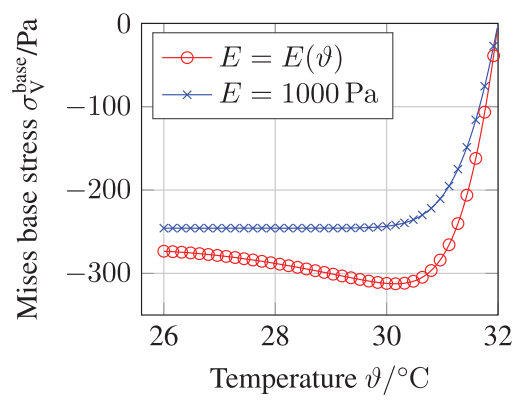

Figure 8. Evolution of the base stress $\sigma_{V}^{\text {base }}$ inside the hydrogel layer over the temperature for temperature-dependent and constant elastic modulus. Please note that only the swelling direction (according to Figure 4(a)), which leads to the closing of pores, is depicted.

captured using the TEM described in the "TEM for active behavior" section.

3.I.I. Base setup. In order to acquire the base setup (see the "Determination of the minimum pore distance" section), that is, the stress distribution of the active three-layer system without pore structures, the CLT (see Appendix 1) is used. Preliminary studies with a 3D finite element model show that the stress state in the three layers is adequately represented by the CLT. The stress due to constrained swelling is constant inside the hydrogel layer. The base setup of the trilayer system can be calculated analytically for the temperature range as described in the previous section. Results of the Mises stress inside the hydrogel layer for different temperatures are depicted in Figure 8. For comparison, the stress evolution is also depicted for a constant mean Young's modulus.

From the diagram, it can be seen that for temperature-dependent Young's modulus $E=E(\vartheta)$, the compressive stress in the hydrogel layers due to swelling increases until $\vartheta \approx 30.5^{\circ} \mathrm{C}$. Then, it diminishes again due to the change of elastic modulus (see Figure 4(b)). The nonlinear behavior stems from the nonlinear swelling behavior of PNiPAAm (see Figure 4(a)). It was verified that the base stress is constant over the 


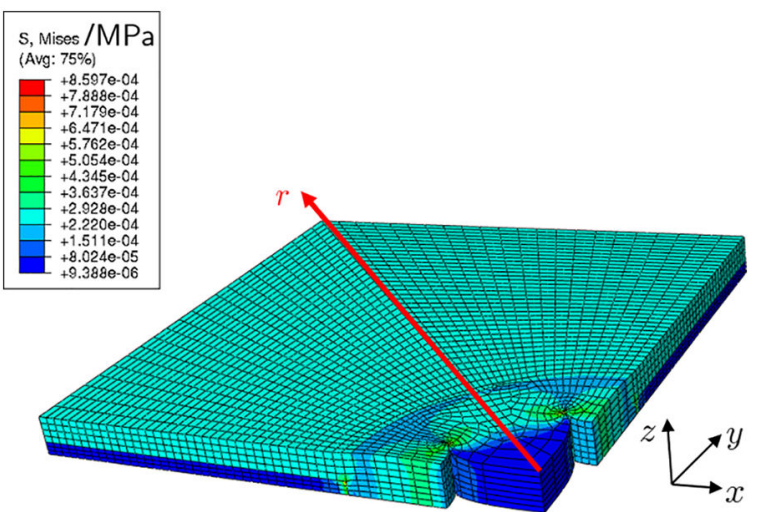

Figure 9. Simulation result of the perforated setup at $\vartheta=26^{\circ} \mathrm{C}$, that is, the completely swollen state with the closed pore. Von Mises stresses are depicted in MPa.

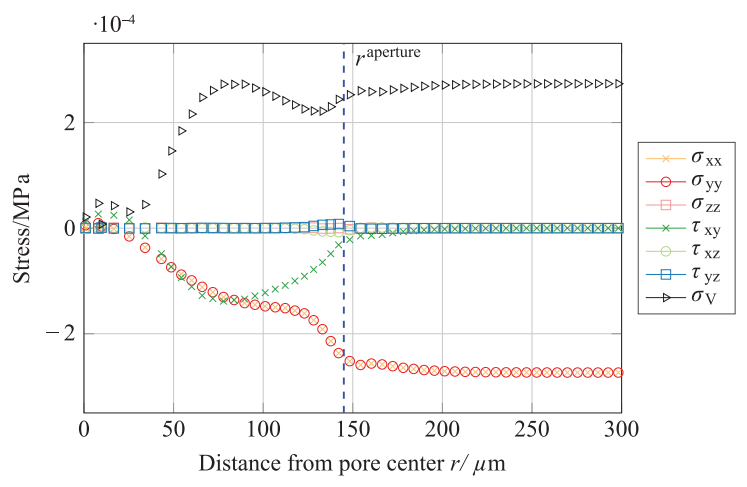

Figure 10. Components of the stress tensor in r-direction for $\vartheta=26^{\circ} \mathrm{C}$. Here, the Mises comparative stress is an adequate measure to capture the approach to a stable end value of the stress components.

thickness direction of the plate using an adequately meshed finite element setup.

3.1.2. Perforated setup. The simulation results for the setup from Figure 7 with respective boundary conditions are depicted in Figure 9. A total of 14,121 quadratic elements of the type C3D20RH were used together with 165 wedge elements of the type C3D15H inside the corners of the cross-structure to avoid excessive distortion of the elements.

In Figure 10, the distribution of different stress measures along the diagonal $r$-direction (see Figure 5(b)) is shown. It can be seen that the Mises stress gives an adequate measure of the approach to a stable end value for all stress distributions.

Using the above explained Mises stress for different temperature steps during the closing cycle of the switchable pore, Figure 11 can be obtained. For $\vartheta=26^{\circ} \mathrm{C}$, the respective base stress $\sigma_{V}^{\text {base }}$ (obtained through CLT,

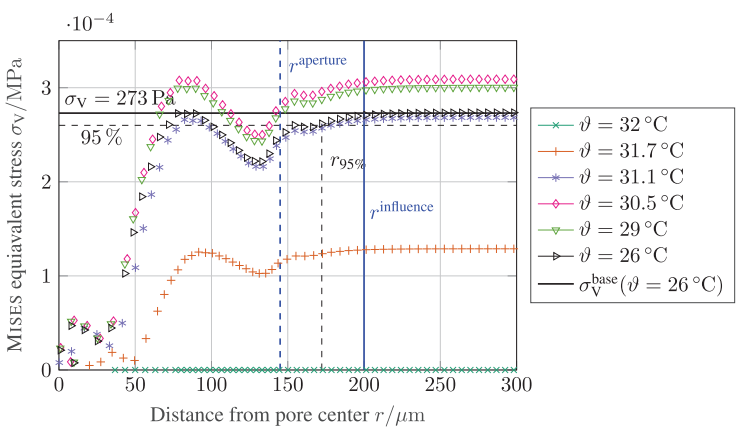

Figure I I. Stress distribution in r-direction for different temperatures. For $\vartheta=26^{\circ} \mathrm{C}$, the stress of the base setup is inserted as $\sigma_{V}$. The intersection with the $95 \%$ value is considered as the point of decayed influence. As influence radius for design purpose, $r^{\text {influence }}=200 \mu \mathrm{m}$ is chosen.

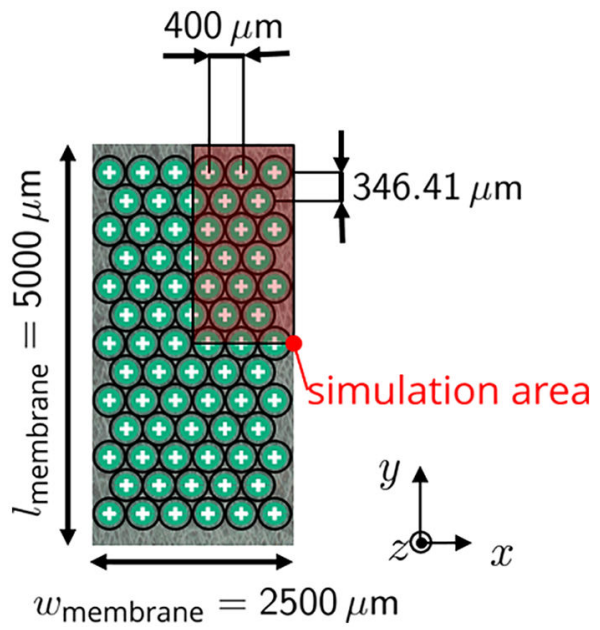

Figure 12. Setup of the permeation control membrane with dense hexagonal pore pattern. With the influence area derived above, a total number of $N^{\text {Pores }}=72$ pores can be realized.

see Appendix 1) is depicted for comparison. From these simulation results, the $95 \%$ value of $\sigma_{V}$ and the respective distance $r^{\text {influence }}$ can be derived.

With reasonable safety-for example, to overcome small tolerances in fabrication $-r^{\text {influence }} \approx 200 \mu \mathrm{m}$ can be assumed. The radius of stress decay - that is, until the base stress $\sigma_{V}^{\text {base }}$ is reached - is valid for every temperature step during the closing of the pore.

With the determined influence radius of $r^{\text {influence }}=200 \mu \mathrm{m}$, the permeation control membrane in our microfluidic test setup can be designed (see Figure 12). The distance between the pores is $d^{\text {pores }}=2 r^{\text {influence }}=400 \mu \mathrm{m}$ (see Figure 5(a)). The hexagonal pattern is chosen according to the "Pore patterns" section.

This setup and the highlighted simulation area are used in the following section to simulate the bending 


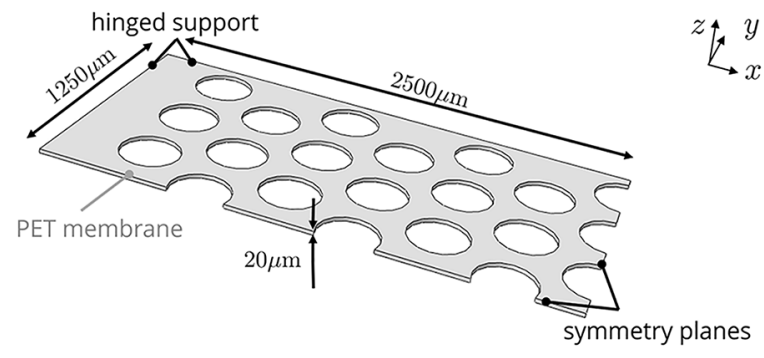

Figure 13. Simulation model of the perforated membrane as simplified membrane setup.

behavior of the permeation control membrane under microfluidic pressure.

\subsection{Simulation results of the membrane bending}

After determination of the actual membrane design in the previous section, now the bending under microfluidic pressure can be simulated. This is needed to ensure that the weakening effect of the pores does not lead to a too large displacement of the membrane. A displacement is considered too large when it leads to a change of the pore shape due to the stretching of the displacement line, which in turn would make the actuation of the pores less precise.

In order to obtain the maximum deformation, the simulation model of the perforated backbone membrane in accordance to the "Bending of the active composite system" section is depicted in Figure 13.

In our simulation, the membrane is subjected to a microfluidic pressure difference of $\Delta p=12 \mathrm{~Pa}$. This was obtained from our previous work (Ehrenhofer and Wallmersperger, 2017a). The pressure correction according to equation (8) leads to a corrected pressure of $\Delta p^{\text {corrected }}=19.37 \mathrm{~Pa}$. The results are depicted in Figure 14. Please note that the deflection of the membrane is very small; the displacements are therefore magnified by a factor of 200 .

The current simulations have shown that even though the membrane is maximally weakened - that is, the maximum number of pores possible without crossinfluence is inserted - only very small displacements occur. The maximum displacement can be found in the middle of the membrane and is $u_{z}=-1.62 \mu \mathrm{m}$.

\section{Conclusion and outlook}

In the present work, we have shown simulation results that support the assumption that through massive parallelization of pores in a permeation control membrane, native blood samples can be handled. The goal of the microfluidic setup - the label-free measurement of the cell size distribution - can thus be achieved.

We have shown that in the design of active composite systems like the permeation control membrane, the area of influence of the active parts has to be considered. This influence region was obtained through the comparison of a base setup and the perforated setup. With known influence regions of a pore, a dense hexagonal pattern was derived. The weakening of the membrane due to perforation was simulated. We found out that this weakening effect is negligible so that the membrane does not lose its functionality in the microfluidic setup.

The mechanical behavior of the composite system was investigated by means of modeling and simulation using the Temperature Expansion Model for the active behavior. This model was used both in the commercial finite element tool Abaqus and in the Classical Laminate Plate Theory.

Besides the analysis of the actual planar membrane system described in this work, the presented strategies allow the design of arbitrary active membrane systems. Especially 3D setups in rolled-up form-known, for example, from electro-chemical condensers - can be

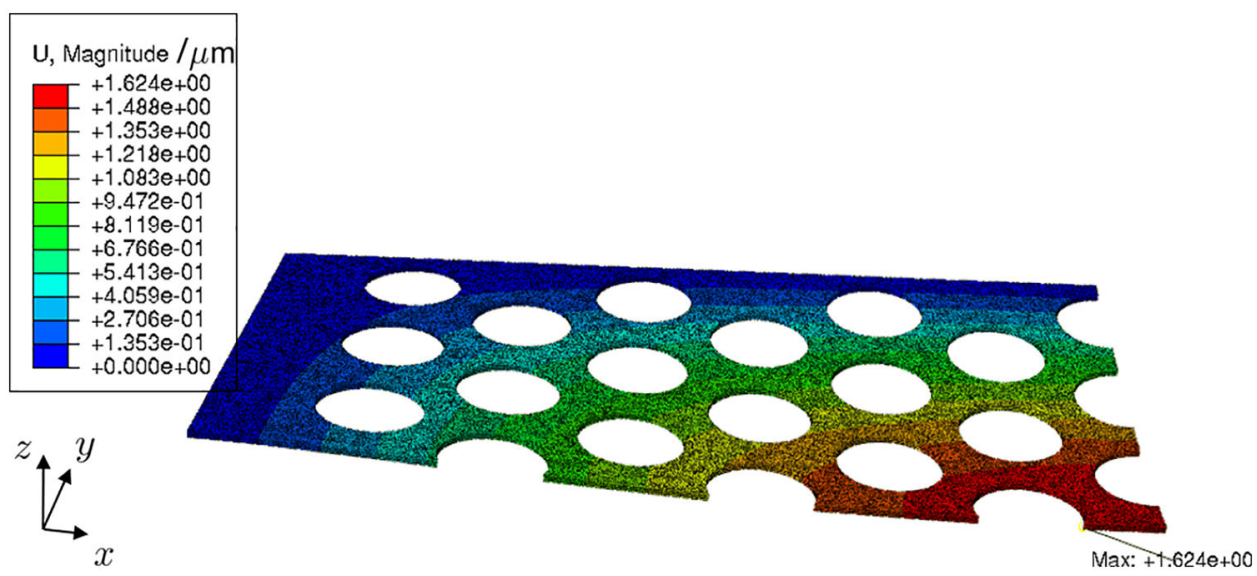

Figure 14. Simulation results for the perforated membrane under uniform microfluidic pressure. The visible displacements in micrometer are magnified by a factor of 200 . 
simulated and assessed in this way. Optimization of the microfluidic measurement setup for the blood cell size distributions allows the design of an autonomous and portable blood analysis device. Such a device can then be used to obtain a patient's blood count in places with limited access to clinical laboratories.

\section{Declaration of conflicting interests}

The author(s) declared no potential conflicts of interest with respect to the research, authorship, and/or publication of this article.

\section{Funding}

The author(s) disclosed receipt of the following financial support for the research, authorship, and/or publication of this article: The present work is supported by the Deutsche Forschungsgemeinschaft (DFG) in the framework of the research training group "Hydrogel-based microsystems" DFG-GRK 1865 at Technische Universität Dresden. It is also supported through the DFG project grant W2323/15-1.

\section{ORCID iD}

Adrian Ehrenhofer (ID) https://orcid.org/0000-0002-2370-8381

\section{References}

Acartürk AY (2009) Simulation of charged hydrated porous materials. PhD Thesis, Universität Stuttgart, Stuttgart.

Adrus N and Ulbricht M (2012) Novel hydrogel pore-filled composite membranes with tunable and temperatureresponsive size-selectivity. Journal of Materials Chemistry 22: 3088-3098.

Anseth KS, Bowman CN and Brannon-Peppas L (1996) Mechanical properties of hydrogels and their experimental determination. Biomaterials 17(17): 1647-1657.

Attaran A, Brummund J and Wallmersperger T (2015) Modeling and simulation of the bending behavior of electrically-stimulated cantilevered hydrogels. Smart Materials and Structures 24(3): 035021.

Binder S, Krause AT, Voit B, et al. (2017) Bisensitive hydrogel with volume compensation properties for force compensation sensors. IEEE Sensors Letters 1(6): 4501004.

Bluhm J, Serdas S and Schröder J (2016) Theoretical framework of modeling of ionic EAPs within the theory of porous media. Archive of Applied Mechanics 86(1-2): 3-19.

Borchardt-Ott W (2009) Die 7 Kristallsysteme. In: BorchardtOtt W (ed.) Kristallographie. Berlin; Heidelberg: Springer, pp. 121-123.

Chang HC and Wang LC (2010) A simple proof of Thue's theorem on circle packing. Available at: https://arxiv.org/ abs/ 1009.4322

Ehlers W (1996) Grundlegende Konzepte in der Theorie Poröser Medien. Technische Mechanik 16: 63-76.

Ehrenhofer A and Wallmersperger T (2017a) Adjustable fluid and particle permeation through hydrogel composite membranes. Journal of Intelligent Material Systems and Structures 29(3): 310-322.
Ehrenhofer A and Wallmersperger T (2017b) Hydrogels for engineering: normalization of swelling due to arbitrary stimulus. In: Proceedings of the SPIE 10163, electroactive polymer actuators and devices XIX, vol. 10163, Portland, OR, 25-29 March, pp. 11-15. Bellingham, WA: SPIE.

Ehrenhofer A and Wallmersperger T (2018) A normalization concept for smart material actuation by the example of hydrogels. Proceedings in Applied Mathematics and Mechanics 18: e201800176.

Ehrenhofer A, Bingel G, Paschew G, et al. (2016a) Permeation control in hydrogel-layered patterned PET membranes with defined switchable pore geometryexperiments and numerical simulation. Sensors and Actuators B: Chemical 232: 499-505.

Ehrenhofer A, Elstner M and Wallmersperger T (2018) Normalization of hydrogel swelling behavior for sensoric and actuatoric applications. Sensors and Actuators B: Chemical 255: 1343-1353.

Ehrenhofer A, Wallmersperger $\mathrm{T}$ and Richter A (2016b) Simulation of controllable permeation in PNIPAAm coated membranes. In: Proceedings of the SPIE 9800, behavior and mechanics of multifunctional materials and composites 2016, vol. 980016, Las Vegas, NV, 20-24 March, pp. 1-13. Bellingham, WA: SPIE.

Forest S (2002) Homogenization methods and mechanics of generalized continua-part 2. Theoretical and Applied Mechanics 28-29: 113-144.

Galante S, Lucantonio A and Nardinocchi P (2013) The multiplicative decomposition of the deformation gradient in the multiphysics modeling of ionic polymers. International Journal of Non-Linear Mechanics 51: 112-120.

Gerlach G and Arndt KF (2009) Hydrogel Sensors and Actuators: Engineering and Technology, vol. 6. Berlin: Springer.

Goldberg M (1970) The packing of equal circles in a square. Mathematics Magazine 43(1): 24-30.

Graham RL and Lubachevsky BD (1996) Repeated patterns of dense packings of equal disks in a square. The Electronic Journal of Combinatorics 3: R16.

Jelkmann W (2011) Physiologie des Menschen. Berlin; Heidelberg: Springer.

Kang MK and Huang R (2011) Swelling-induced instability of substrate-attached hydrogel lines. International Journal of Applied Mechanics 3(2): 219-233.

Leichsenring P and Wallmersperger T (2017) Modeling and simulation of the chemically induced swelling behavior of anionic polyelectrolyte gels by applying the theory of porous media. Smart Materials and Structures 26(3): 035007.

Li H (2010) Smart Hydrogel Modelling. Berlin: Springer Science + Business Media.

Mota Soares CA, Mota Soares CM and Freitas MJ (1999) Mechanics of Composite Materials and Structures, vol. 361. Berlin: Springer Science + Business Media.

Reddy JN (2004) Mechanics of Laminated Composite Plates and Shells: Theory and Analysis. Boca Raton, FL: CRC Press.

Seuss M, Schmolke W, Drechsler A, et al. (2016) Core-shell microgels with switchable elasticity at constant interfacial interaction. ACS Applied Materials \& Interfaces 8(25): 16317-16327.

Shields CW, Reyes CD and López GP (2015) Microfluidic cell sorting: a review of the advances in the separation of cells 
from debulking to rare cell isolation. Lab on a Chip 15(5): $1230-1249$.

Sobczyk M and Wallmersperger $\mathrm{T}$ (2016) Modeling and simulation of the electro-chemical behavior of chemically stimulated polyelectrolyte hydrogel layer composites. Journal of Intelligent Material Systems and Structures 27(13): 1725-1737.

Sun T and Qing G (2011) Biomimetic smart interface materials for biological applications. Advanced Materials 23(12): H57-H77.

Urayama K and Takigawa T (2012) Volume of polymer gels coupled to deformation. Soft Matter 31(8): 8017-8029.

Wallmersperger T, Attaran A, Keller K, et al. (2013) Modeling and simulation of hydrogels for the application as bending actuators. In: Sadowski G and Richtering W (eds) Intelligent Hydrogels (Progress in Colloid and Polymer Science), vol. 140. New York: Springer, pp. 189-204.

\section{Appendix I}

\section{Classical Laminate Theory for the derivation of the base setup}

The main idea of the Classical Laminate Theory (CLT) is the combination of a stack of lamina with individual bending and tensile stiffness (Reddy, 2004; Mota Soares et al., 1999). The layering of lamina provides the composite system with its overall mechanical abilities. The coordinate definition can be found in Figure 3.

Please note that due to symmetry considerations, the Voigt notation is used henceforth for the mechanical tensor relations. This allows an easier representation using matrices (double underlined, for example, $\underline{\underline{E} \text { ) and }}$ vectors (single underlined, for example, $\underline{\alpha}$ ).

The mathematical problem to obtain strain and flexion from given forces and moments is

$$
\left(\begin{array}{l}
\underline{n} \\
\underline{m}
\end{array}\right)=\left(\begin{array}{ll}
\underline{\underline{A}} & \underline{\underline{B}} \\
\underline{\underline{B}} & \underline{\underline{D}}
\end{array}\right)\left(\begin{array}{l}
\underline{\varepsilon} \\
\underline{\kappa}
\end{array}\right)-\Delta T\left(\begin{array}{l}
\underline{\underline{A}} \underline{\alpha} \\
\underline{\underline{B}} \underline{\alpha}
\end{array}\right)
$$

Here, $\underline{n}=\left(n_{x}, n_{y}, n_{x y}\right)$ and $\underline{m}=\left(m_{x}, m_{y}, m_{x y}\right)$ are the force and moment resultants of the laminate setup. The thermal expansion coefficients are $\underline{\alpha}$ and can be applied in the framework of the Temperature Expansion Model (TEM), described in the "TEM for active behavior" section. The resultants and expansion terms for small deformations lead to the elastic strains

$$
\underline{\varepsilon}^{\text {elastic }}=\left(\begin{array}{c}
\varepsilon_{x x}-\varepsilon_{\mathrm{Tx}} \\
\varepsilon_{y y}-\varepsilon_{\mathrm{Ty}} \\
\varepsilon_{x y}-\varepsilon_{\mathrm{Txy}}
\end{array}\right)=\left(\begin{array}{c}
\frac{\partial u_{x}}{\partial x}-\alpha_{x} \Delta T \\
\frac{\partial u_{y}}{\partial y}-\alpha_{y} \Delta T \\
\frac{1}{2}\left(\left(\frac{\partial u_{x}}{\partial y}+\frac{\partial u_{y}}{\partial x}\right)-\alpha_{x y} \Delta T\right)
\end{array}\right)
$$

and to curvatures (without temperature components due to constant temperature over the height)

$$
\underline{\kappa}=\left(\begin{array}{c}
\kappa_{x x} \\
\kappa_{y y} \\
\kappa_{x y}
\end{array}\right)=\left(\begin{array}{c}
-\frac{\partial^{2} u_{z}}{\partial x^{2}} \\
-\frac{\partial^{2} u_{z}}{\partial y^{2}} \\
-\frac{\partial^{2} u_{z}}{\partial x \partial y}
\end{array}\right)
$$

The stiffness matrix of the laminate is composed of the linear stiffness matrix $\underline{\underline{A}}$, the bending stiffness matrix $\underline{D}$, and a coupling matrix $\underline{\bar{B}}$.

Without the coupling matrix $\underline{\underline{B}}$ that is zero due to symmetry of the setup, the problem dissolves into two separate systems of equations. They are the membrane and the plate state.

\section{Membrane state/tensional stiffness}

In the mechanical sense, a membrane can only be subject to linear stretch or pressing; it is therefore a $2 \mathrm{D}$ stress state. To derive $\underline{\underline{A}}_{\text {total }}$, the thickness $t_{k}$ and matrix of elastic moduli $\underline{\underline{E}}_{k}$ of every layer are used

$$
\underline{\underline{A}}_{\mathrm{total}}=\sum_{k} \underline{\underline{E}}_{k} t_{k}
$$

The elasticity matrix for every layer $k$ is given as

$$
\underline{E}_{k}=\left(\begin{array}{ccc}
E_{11} & E_{12} & E_{16} \\
E_{21} & E_{22} & E_{26} \\
E_{61} & E_{62} & E_{66}
\end{array}\right)_{k}
$$

Here, the parameters are transformed from the local coordinates of a layer. However, since the material values in the current case are not direction-dependent, the transformation relations are not needed.

\section{Plate state}

For the plate state, the single layers are each considered with two parts: (I) their lever to the neutral axis and (II) their original bending stiffness. This leads to the derived bending stiffness of

$$
\underline{\underline{D}}=\sum_{k} \frac{1}{3}\left(z_{k}^{3}-z_{k-1}^{3}\right) \underline{\underline{E}}_{k}=\sum_{k}(\underbrace{t_{k} z_{m k}^{2}}_{(\mathrm{I})}+\underbrace{\frac{1}{12} t_{k}^{3}}_{(\mathrm{II})}) \underline{\underline{E}}_{k}
$$

From the derivations shown above we obtain simple equations for membrane state (only stretch) and plate state (only bending). For the trilayer system (Figure 3) described above, the stiffness of the membrane state 
(equation (12)) drastically simplifies. Due to the isotropic behavior, no direction transformation is necessary. For every single layer, the stiffness is added due to parallel setup

$$
\underline{\underline{A}}_{\mathrm{total}}=2 t_{\mathrm{HG}} \underline{\underline{E}}_{\mathrm{HG}}+t_{\mathrm{PET}} \underline{\underline{\mathrm{E}}}_{\mathrm{PET}}
$$

where the subscript HG denotes the hydrogel and PET (polyethylene terephthalate) the backbone membrane, and $t$ is the thickness of the layer according to Figure 3. The bending stiffness for our system (equation (14)) is

$$
\begin{aligned}
& \underline{\underline{D}}_{\mathrm{total}}= \\
& \quad 2\left(t_{\mathrm{HG}}\left(\frac{t_{\mathrm{PET}}+t_{\mathrm{HG}}}{2}\right)^{2}+\frac{1}{12} t_{\mathrm{HG}}^{3}\right) \underline{\underline{E}}_{\mathrm{HG}}+\frac{1}{12} t_{\mathrm{PET}}^{3} \underline{\underline{E}}_{\mathrm{PET}}
\end{aligned}
$$

Using the temperature-dependent elastic modulus of the hydrogel, the stresses of the base setup can be obtained analytically. This is done in the "Results and discussion" section. 